\title{
Decentralized Control for Self-driving Cars That can Freely Move on Two-dimensional Plane
}

\author{
Takeshi Kano $^{1}$, Mayuko Iwamoto ${ }^{2}$, Daishin Ueyama ${ }^{3}$ \\ ${ }^{1}$ Research Institute of Electrical Communication, Tohoku University, Sendai, Japan \\ tkano@riec.tohoku.ac.jp \\ ${ }^{2}$ Interdisciplinary Faculty of Science and Engineering, Shimane University, Matsue, Japan \\ miwamoto@riko.shimane-u.ac.jp \\ ${ }^{3}$ Faculty of Engineering, Musashino University, Tokyo, Japan \\ d.ueyama@gmail.com
}

\begin{abstract}
In the current traffic rules, cars have to move along lanes and to stop at red traffic lights. However, in the future when all cars become completely driverless, these traffic rules may vanish and cars may be allowed to move freely on two-dimensional plane by avoiding others like pedestrian flow. This innovation could greatly reduce traffic jams. In this study, we propose a decentralized control scheme for future self-driving cars that can freely move on two-dimensional plane, based on the social force model widely used as the model of pedestrian flow. The performance of the proposed scheme is validated via simulation. Although this study is still conceptual and does not consider realistic details, we believe that it paves the way to developing novel traffic systems.
\end{abstract}

Keywords: Decentralized control, Self-driving cars, Social force model, Pedestrian flow

\section{Introduction}

Technologies for self-driving cars have been developed within the framework of the conventional traffic rules in which cars have to move along lanes and to stop at red traffic lights. However, these traffic rules, which limit traffic flow and often cause traffic jams, may not be necessary in the future when all cars become completely driverless, because self-driving cars will be able to move more accurately than cars driven by humans. It is expected that traffic jams will be greatly reduced if self-driving cars move freely on two-dimensional plane by avoiding others like pedestrian flow.

In this study, we propose a decentralized control scheme for future self-driving cars that can freely move on two-dimensional plane. Because this study looks ahead to distant future, we employ minimal assumptions, rather than consider realistic and detailed problems, to capture the essence of the control. Social force model [1,2], a simple model of pedestrian flow, would be a suitable platform for considering this problem. We construct the control scheme by improving the social force model and demonstrate that the simulated self-driving cars can move fast, smoothly, and safely.

\section{Model}

In the social force model [1,2], each pedestrian on a two-dimensional plane is regarded as a circular particle with the radius of $r$, and its time evolution is described as

$m \ddot{\mathbf{x}}_{i}=a\left(v_{0} \mathbf{e}_{i}-\dot{\mathbf{x}}_{i}\right)+\sum_{j \neq i}\left(\mathbf{f}_{i j}^{\text {phys }}+\mathbf{f}_{i j}^{\text {soc }}\right)+\sum_{b}\left(\mathbf{f}_{i b}^{\text {phys }}+\mathbf{f}_{i b}^{\mathrm{soc}}\right)$,

where $m$ is the mass of the particle, $\mathbf{x}_{i}$ is the position of the particle $i, \mathbf{e}_{i}$ is the unit vector that represents the direction particle $i$ wants to move, $v_{0}$ is the target speed, and $a$ is a positive constant. The second and third terms on the right-hand side denote forces generated by the interaction with the other particles and that with the environment, e.g., walls, respectively. Superscripts "phys" and "soc" for these forces denote physical and social forces, respectively. Details are provided in [1,2].

The model of self-driving cars proposed here is also described in the same form as Eq. (1). Here, whereas the social force originates from psychological effect, i.e., desire to avoid others, in the case of pedestrian flow [1,2], it is redefined as the control input to self-driving cars and is designed based on the 
prediction of future motion of itself and nearby cars (see Steps $1-4$ below). Note that the basic idea of the design is similar to our previous work on the decentralized control of traffic signals [3].

The social force (control input) $\mathbf{f}_{i j}^{\text {soc }}$ in the proposed model is calculated according to the following 4 steps $\left(\mathbf{f}_{i b}^{\text {soc }}\right.$ can be calculated in a similar manner as $\left.\mathbf{f}_{i j}^{\text {Soc }}\right)$ :

Step 1: Each car detects the relative position and velocity of cars within the distance $R$ from itself every time interval $\Delta t$.

Step 2: Based on the information obtained in Step 1, each car predicts the future motion of cars within the distance $R$ from itself. Because the interaction terms (the second and third terms on the right-hand side) in Eq. (1) are hard to predict, the equation in which the interaction terms are omitted from Eq. (1), $m \ddot{\mathbf{x}}_{i}=a\left(v_{0} \mathbf{e}_{i}-\dot{\mathbf{x}}_{i}\right)$,

is solved numerically for duration $T$, wherein the initial position and velocity of the cars are the same as those obtained in Step 1 (Fig. 1).

Step 3: Based on the numerical calculation performed in Step 2, the expected time until the distance between cars $i$ and $j$ takes a minimum value or until car $j$ collides car $i, \tau_{i j}$, is derived (Fig. 1). Here, $\tau_{i j}=0$ when the distance is expected to increase monotonically, and $\tau_{i j}=' I$ ' when the distance is expected to decrease monotonically without any collision. Further, the expected distance between cars $i$ and $j$ after the time interval $\tau_{i j}$, $\hat{d}_{i j}\left(\tau_{i j}\right)$, is also derived.

Step 4: The social force $\mathbf{f}_{i j}^{\text {soc }}$ is derived according to the following equation:

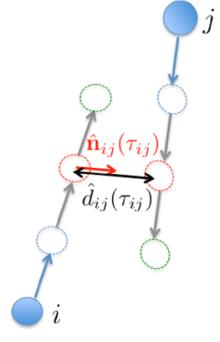

(a)

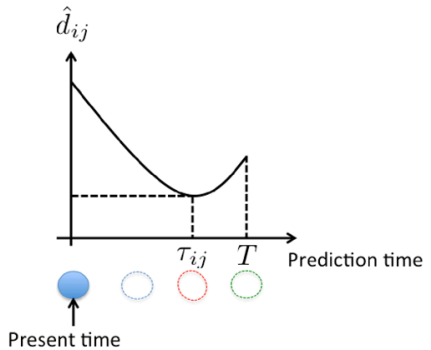

(b)

Fig. 1: Schematic of the proposed control scheme. (a) Based on the present position and velocity of the cars (blue filled circles and arrows, respectively), their future trajectories are estimated by calculating Eq. (2)) (blue, red, and green dashed circles). (b) Schematic of the time evolution of the distance between cars $i$ and $j$ for the situation shown in (a).

$\mathbf{f}_{i j}^{\text {soc }}=-A \exp \left(-\frac{d_{i j}-2 r}{B}\right) \mathbf{n}_{i j}-C\left(1-\frac{\tau_{i j}}{T}\right) \hat{d}_{i j}\left(\tau_{i j}\right)^{-\mu} \hat{\mathbf{n}}_{i j}\left(\tau_{i j}\right)$,

where $A, B, C$, and $\mu$ are positive constants, and $d_{i j}$ is the distance between cars $i$ and $j$. The unit vectors $\mathbf{n}_{i j}$ and $\hat{\mathbf{n}}_{i j}\left(\tau_{i j}\right)$ are given by

$$
\begin{gathered}
\mathbf{n}_{i j}=\frac{\mathbf{x}_{j}-\mathbf{x}_{i}}{\left|\mathbf{x}_{j}-\mathbf{x}_{i}\right|}, \\
\hat{\mathbf{n}}_{i j}=\frac{\hat{\mathbf{x}}_{j}\left(\tau_{i j}\right)-\hat{\mathbf{x}}_{i}\left(\tau_{i j}\right)}{\left|\hat{\mathbf{x}}_{j}\left(\tau_{i j}\right)-\hat{\mathbf{x}}_{i}\left(\tau_{i j}\right)\right|},
\end{gathered}
$$

where $\hat{\mathbf{x}}_{i}\left(\tau_{i j}\right)$ is the expected position of car $i$ after the time interval $\tau_{i j}$ (Fig. 1). The first and second terms on the right-hand side in Eq. (3) denote the exclusive volume effect and the avoidance force based on the prediction, respectively. The term $\left(1-\tau_{i j} / T\right) \hat{d}_{i j}\left(\tau_{i j}\right)^{-\mu}$ in Eq. (3) was introduced because the avoidance force should be large when the distance between cars $i$ and $j$ is expected to become small in near future. Note that the proposed model is consistent with the original social force model $[1,2]$ when $A>0$ and $C=0$.

\section{Simulation}

Simulation of the proposed model was performed. The deflection angle of $\mathbf{e}_{i}$ was set to be random and did not vary during the simulation. The periodic boundary condition was employed. The performance was evaluated by the following indices $E_{1}, E_{2}$, and $E_{3}$ : 


$$
\begin{aligned}
& E_{1}=\frac{\sum_{k=1}^{n} \sum_{i=1}^{N}\left(\dot{\mathbf{x}}_{i}(k) \cdot \mathbf{e}_{i}\right)}{v_{0} n N}, \\
& E_{2}=\frac{\sum_{k=1}^{n} \sum_{i=1}^{N}\left|\ddot{\mathbf{x}}_{i}(k)\right|^{2}}{n N}, \\
& E_{3}=\frac{\sum_{k=1}^{n} \sum_{i=1}^{N}\left|\mathbf{f}_{i j}^{\text {phys }}(k)\right|}{n N},
\end{aligned}
$$

where $k$ is the time step, $n$ is the maximal time step, and $N$ is the number of particles. Index $E_{1}$ is large when cars move fast in the direction they want to move, while indices $E_{2}$ and $E_{3}$ are small when cars move smoothly and safely, respectively.

The result when parameters $A$ and $C$ are varied with the other parameters fixed is shown in Fig. 2. It is found that $E_{1}$ increases as $A$ decreases and $C$ increases (Fig. 2(a)), while $E_{3}$ decreases as $A$ and $C$ increase (Fig. 2(c)). In contrast, $E_{2}$ is small in the area where $A$ is small and $C$ is around 3.0 $\left[\mathrm{m}^{4} \mathrm{~kg} \mathrm{~s}^{-2}\right]$.

White and yellow arrows in Fig. 2 point the parameter regions where "speed", "smoothness", and "safety" are well-balanced for the proposed model $(A>0, C>0)$ and for the original social force model $(A>0, C=0)[1,2]$, respectively. It is clear that the performance is better for the proposed model than for the original social force model ( $E_{1}=0.85, E_{2}=140.7\left[\mathrm{~m}^{2} \mathrm{~kg} \mathrm{~s}^{-4}\right]$, and $E_{3}=53.0\left[\mathrm{~m} \mathrm{~kg} \mathrm{~s}^{-2}\right]$ at the region where the white arrows point, while $E_{1}=0.71$, $E_{2}=428.5\left[\mathrm{~m}^{2} \mathrm{~kg} \mathrm{~s}^{-4}\right]$, and $E_{3}=233.0\left[\mathrm{~m} \mathrm{~kg} \mathrm{~s}^{-2}\right]$ and at the region where the yellow arrows point). Thus, the avoidance force based on the prediction, which was newly introduced, plays a crucial role for enabling self-driving cars to move fast, smoothly, and safely.

\section{Conclusion}

We proposed a decentralized control scheme for future self-driving cars that can freely move on two-dimensional plane, based on the social force model. We demonstrated via simulations that cars with the proposed control scheme can move fast, smoothly, and safely. It should be noted that this
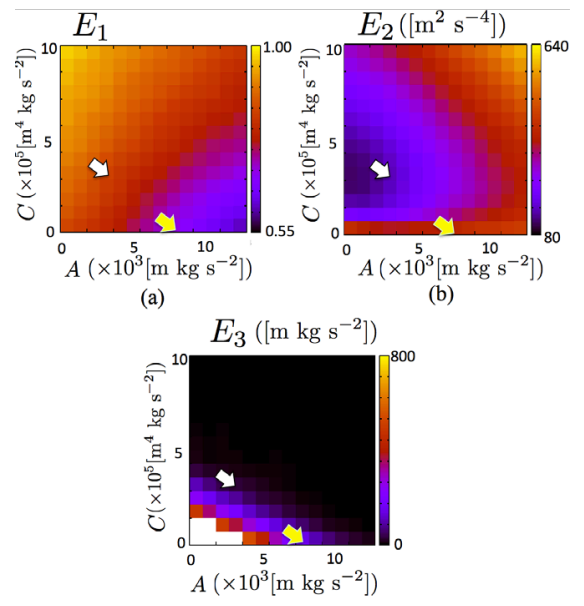

Fig. 2: Values of $E_{1}, \stackrel{(\text { c) }}{E_{2}}$, and $E_{3}$ when parameters $A$ and $C$ are varied are shown in (a), (b), and (c), respectively. White areas in (c) denote the areas in which $E_{3} \geq 800$ [m $\left.\mathrm{kg} \mathrm{s}^{-2}\right]$. Explanations for the white and yellow arrows are provided in the main text. study is still conceptual and does not consider realistic issues (e.g., calibration of parameters to realistic values of current cars etc.). However, we believe that our new concept becomes a breakthrough for developing novel traffic systems. Finally, we would like to note that the model proposed here could be also used as the model of pedestrian flow because pedestrians change their path by predicting the movement of their nearby pedestrians. Hence, we believe that this study contributes to the field of pedestrian and evacuation dynamics.

\section{Acknowledgements}

The authors would like to thank Prof. Akio Ishiguro and Prof. Takashi Akamatsu of Tohoku University for their helpful suggestions.

\section{References}

[1] D. Helbing and P. Molnár, "Social force model for pedestrian dynamics", Phys. Rev. E, vol. 51, pp. 4282-4286, 1995.

[2] D. Helbing, "Traffic and related self-driven many-particle systems", Rev. Mod. Phys., vol. 73, pp. 1067-1141, 2001.

[3] T. Kano, Y. Sugiyama, and A. Ishiguro, "Autonomous decentralized control of traffic signals that can adapt to changes in traffic", Collective Dynamics, vol. 1, A5, pp. 1-18, 2016. 